\title{
Fexofenadine HCl Microspheres - Can it be the First Line therapy for Allergic Disorders?
}

\section{Paroma Arefin $^{1,2}$ *, Md Shehan Habib ${ }^{1}$, Mohammad Mostafa ${ }^{1}$, Dipankar Chakraborty ${ }^{1}$, Sreebash Chandra Bhattacharjee ${ }^{1}$, Md Saidul Arefin ${ }^{3}$ and Debabrata Karmakar ${ }^{4}$}

\author{
${ }^{1}$ BCSIR Chattogram Laboratories, Bangladesh Council of Scientific \\ and Industrial Research, Bangladesh. \\ ${ }^{2}$ Institute of Food Science and Technology, Bangladesh Council of Scientific \\ and Industrial Research, Bangladesh. \\ ${ }^{3}$ Institute of Nutrition and Food Science, University of Dhaka, Dhaka-1000, Bangladesh. \\ ${ }^{4}$ Institute of Technology Transfer and Innovation, Bangladesh Council of \\ Scientific and Industrial Research, Bangladesh. \\ http://dx.doi.org/10.13005/bbra/2961
}

(Received: 04 October 2021; accepted: 24 December 2021)

Fexofenadine $\mathrm{HCl}$ is a second-generation antihistamine which is commonly used for allergic disorders. But it has low bioavailability. Intranasal corticosteroids (INCs) and Immunotherapy and Allergen Specific Immunotherapy (ASIT) are now commonly being suggested for the treatment of allergic disorders. Despite the fact that current treatment alternatives have been in use for decades, patient quality of life has remained static. The treatment options are not much explored for their respective adverse effects. Therefore, they are in desperate need of research. Fexofenadine $\mathrm{HCl}$ is available in the form of a suspension, tablet, or capsule. In our current study, we have explored whether microspheres can be the perfect dosage form of Fexofenadine HCl to treat allergic disorders considering the pharmacokinetics of the drug, available dosage forms options and the probable side effects of the current therapies.

Keywords: Allergen Specific Immunotherapy (ASIT), Allergic disorder, Fexofenadine $\mathrm{HCl}$, Half-life, Intranasal corticosteroids (INCs), Microsphere.

Antihistamines of the first generation were formerly used to treat allergic disorders. ${ }^{1,2}$ Secondgeneration antihistamines, such as Terfenadine, Fexofenadine $\mathrm{HCl}$ etc were next being prescribed owing to avoid the sedative side effects observed with first generation drugs. ${ }^{3-5}$ Terfenadine's carboxylic acid metabolite is Fexofendine $\mathrm{HCl}$. There are no sedative impacts of fexofenadine $\mathrm{HCl}{ }^{2,6,7}$ It has no sedative or electrocardiographic effects, according to reports. Fexofenadine $\mathrm{HCl}$ is available in pill, capsule, and oral suspension dosage forms. It has been established that they are bioequivalent. ${ }^{6,8}$ Fexofenadine is quickly absorbed after consumption and has a lengthy duration of action (half-life 14.4 hours), making it suitable for once-daily use. Adults and children over the age of 12 should take $60 \mathrm{mg}$ twice a day, $120 \mathrm{mg}$ once a day, or $180 \mathrm{mg}$ orally once a day of fexofenadine 
$\mathrm{HCl}$. The suggested dose for children aged 6 to 11 years is $30 \mathrm{mg}$ twice day. ${ }^{10-12}$ Oral suspension is indicated for children aged six months and above, with a suggested dose of $15 \mathrm{mg}$ twice day for children aged six months to two years and 30 mg twice daily for children aged two to eleven years. ${ }^{12,13}$

In our study, we suggest that microspheres can be the perfect dosage form of Fexofenadine $\mathrm{HCl}$ to treat allergic disorders. In microcapsules, the active drug molecule is encapsulated and enclosed by a distinct polymeric wall, while in microspheres, the drug is evenly diffused all through the polymeric material. ${ }^{10,13}$ Microspheres provide a variety of benefits over traditional drug delivery systems, including the ability to transport therapeutic active ingredients to the region of interest of the body in a regulated and sustained way, resulting in better therapeutic effects. Microspheres are novel drug delivery systems with particle sizes 1-1000im. ${ }^{14}$ Conventional sustained release dosage forms like tablets and capsules have the risk of dose dumping. ${ }^{15}$ Besides, the drugs which has lower bioavailability are eliminated from the physiological system with lower degree of absorption. ${ }^{16,17}$ Thus the total drug administered is not utilized, and the drug passes through the metabolism organs like kidney or liver and have harmful effects. ${ }^{18}$ With the use of sustained release microspheres, we can avoid dose dumping risks and thus increase safety profile. We will need lower dose than the conventional ones to reach the minimum effective concentration (MIC). ${ }^{19}$ Fexofenadine $\mathrm{HCl}$ microspheres is suitable for making the best use of the given dose with less side effects.

Comparison of Current Treatment Approaches

One of the most frequent kinds of allergies is allergic rhinitis or hay fever. ${ }^{20,21}$ According to recent research, its frequency has risen from 1.4 to 45 percent in the last several decades. ${ }^{7}$ Allergic rhinitis has both direct and indirect consequences on one's quality of life, and it's often paired by asthma, middle ear irritation, nasal polyps, sinusitis, and respiratory tract infections. Allergic disorder is a common condition that affects millions of people in the world. ${ }^{22,23}$ For moderate to severe allergic condition, intranasal corticosteroids (INCs) are frequently recommended as first-line treatment. Beclomethasone, budesonide, ciclesonide, flunisolide, fluticasone furoate, fluticasone propionate, mometasone, and triamcinolone are among the most commonly used INCs. ${ }^{24}$ Their anti-inflammatory properties reduce inflammation, edema, and vascular leakage, which helps to relieve rhinorrhea and congestion. ${ }^{25-27}$ INCs reduce the amount of histamine-producing mast cells in the nasal mucosa, which reduces itchiness and sneezing. INCS has longer onset of action. ${ }^{28,29}$ Although these medications have comparable safety and effectiveness, systemic steroids usually have major side effects. ${ }^{30-32}$ INCs have a low risk of systemic adverse events (AEs), such as hypothalamic-pituitary-adrenal axis suppression or growth suppression, when administered at prescribed dosages and durations..$^{33,34}$ Researchers are suggesting intranasal corticosteroids (INCs) due to their greater efficacy over antihistamines. ${ }^{33,35,36}$

While not a cure, Allergen-Specific Immunotherapy (ASIT) remains the only treatment that can modify the natural course of an allergic illness. But the danger of allergic immunotherapyrelated systemic responses (SRs), which are estimated to occur in $1 \%$ to $4 \%$ of patients and can vary from moderate to lethal in severity, is a significant obstacle to applying this unique and successful therapeutic option. $4,7,37,38$

An allergic patient is given a constantly higher dosage of an allergen immunotherapy in order to reduce their symptoms when they come into contact with the allergen they are allergic to. Despite the fact that ASIT is not a remedy, it is the only therapy that might potentially modify the natural path of an allergic condition. ${ }^{27,39}$ For those with atopy, which is characterized as a heightened predisposition toward IgE-based sensitivity, which results in the formation of particular IgE antibodies to common environmental allergens-about $40 \%$ of the overall population is believed to be IgEbased sensitive. ${ }^{20,39}$ The therapeutic arsenal should be expanded to include any treatment that can alleviate symptoms without inflicting intolerable side-effects.

\section{Fexofenadine $\mathrm{HCl}$ microspheres- $\mathrm{Can}$ it be first line therapy?}

But in the perspective of safety, antihistamines are more accepted globally ${ }^{36,40,41}$. So, this is chosen as the first-line therapy. It has also been offered in a sustained release dosage form of tablet or capsule to make the medication dose 
more convenient for patients. Microspheres are used to increase dose efficiency while reducing the likelihood of adverse effects. Pharmaceutical firms may develop the most optimal release patterns using microspheres of active pharmaceutical ingredients (API). ${ }^{14,18}$ Polymeric microspheres containing fexofenadine $\mathrm{HCl}$ may assist achieve the optimum absorption profile and bioavailability. Furthermore, microspheres give a large margin of safety by eliminating the risk of dosage burst. ${ }^{10,13}$ Fexofenadine $\mathrm{HCl}$ is absorbed at a rate of 30 40 percent. The remainder is eliminated via the kidneys without being absorbed. Side effects would be lessened if Fexofenadine $\mathrm{HCl}$ sustained-release microspheres of polymer blends were utilized since less medicine would be supplied and the kidney would clear out less drug. ${ }^{14}$ The needed dosage might be given to the patient based on his or her particular needs, pathophysiology, and physical condition. The needed dosage in microspheres may also be supplied as a capsule, and the dose can be determined based on drug entrapment efficiency and microsphere drug loading. It would also assist to lower manufacturing costs by requiring less medicine to be included into the pharmaceutical formulations. As a consequence, patients are likely to get treatments at a reduced cost. To balance its lengthy half-life and low absorption, the polymer concentration should be maintained moderate. Microspheres dosage forms are coming into light day by day. When drug is a blessing, it can do the most harm to the body. Now-a -days the main challenge is not to find out the drug for a treatment, but the challenge is how to deliver the drug for its maximum safety and effectiveness. Fexofenadine $\mathrm{HCl}$ is obviously safer than steroids and immunotherapy. If we can modify and optimize the dosage form, it can be used as the first line therapy for the allergic disorders.

\section{CONCLUSION}

Effective treatment options for allergic disorders may help the patients achieve a higher quality of life. Researchers are always attempting to identify methods to lessen the side effects of drugs by altering the molecule, site specific targeted medication release, and dosage forms and dosage forms with cost-effectiveness. Due to the reduced effectiveness of Fexofenadine $\mathrm{HCl}$, i.e. its lesser bioavailability, intranasal corticosteroids (INCS), subcutaneous, and sublingual immunotherapy have been recommended as current therapeutic options. If Fexofenadine $\mathrm{HCl}$ efficacy can be increased by boosting bioavailability, it might be the 'state of art' alternative for patients in every way: convenience, cost effectiveness, and a large margin of safety.

\section{ACKNOWLEDGEMENT}

We are grateful to Bangladesh Council of Scientific and Industrial Research.

\section{Conflict of Interest}

This research has no conflict of interest.

\section{Funding Source}

None.

\section{REFERENCES}

1. Simon FER, Simons KJ. H1 Antihistamines: Current Status and Future Directions. World Allergy Organ J. 2008;1(9):145-155. doi:10.1186/1939-4551-1-9-145

2. Fein MN, Fischer DA, O'Keefe AW, Sussman GL. CSACI position statement: Newer generation H1-antihistamines are safer than first-generation H1-antihistamines and should be the first-line antihistamines for the treatment of allergic rhinitis and urticaria. Allergy, Asthma Clin Immunol. 2019;15(1):1-6. doi:10.1186/s13223019-0375-9

3. Randall KL, Hawkins CA. Antihistamines and allergy. Aust Prescr. 2018;41(2):42-45. doi:10.18773/austprescr.2018.013

4. Simons FER, Simons KJ. Histamine and H1antihistamines: Celebrating a century of progress. J Allergy Clin Immunol. 2011;128(6):1139-1150. e4. doi:10.1016/j.jaci.2011.09.005

5. Maeda T, Babazono A, Nishi T. Surveillance of First-Generation H1-Antihistamine Use for Older Patients with Dementia in Japan: A Retrospective Cohort Study. Curr Gerontol Geriatr Res. 2018;2018:1-6. doi:10.1155/2018/3406210

6. Ahn JH, Kim J, Rehman NU, Kim HJ, Ahn MJ, Chung HJ. Effect of rumex acetosa extract, a herbal drug, on the absorption of fexofenadine. Pharmaceutics. 2020;12(6):1-14. doi:10.3390/ pharmaceutics 12060547

7. Slavin RG. Treating rhinitis in the older population: Special considerations. Allergy, Asthma Clin Immunol. 2009;5(1):1-4. doi:10.1186/1710-1492-5-9

8. Bernstein DI, Schoenwetter WF, Nathan RA, Storms W, Ahlbrandt R, Mason J. Efficacy 
and Safety of Fexofenadine Hydrochloride for Treatment of Seasonal Allergic Rhinitis. Ann Allergy, Asthma Immunol. 1997;79(5):443-448. doi:10.1016/S1081-1206(10)63041-4

9. Kaiser HB, Rooklin A, Spangler D, Capano D. Efficacy of Loratadine Compared with Fexofenadine or Placebo for the Treatment of Seasonal Allergic Rhinitis. Clin Drug Investig. 2001;21(8):571-578. doi:10.2165/00044011200121080-00006

10. Arefin P, Hasan I, Reza MS. Design, characterization and in vitro evaluation of HPMC K100 M CR loaded Fexofenadine $\mathrm{HCl}$ microspheres. Springerplus. 2016;5(1). doi:10.1186/s40064-016-2322-2

11. Huh Y, Cho H-J, Yoon I-S, et al. Preparation and evaluation of spray-dried hyaluronic acid microspheres for intranasal delivery of fexofenadine hydrochloride. Eur J Pharm Sci. 2010;40(1):9-15. doi:10.1016/j.ejps.2010.02.002

12. Shasho H, Sakur AA, Trefi S. Separation and assay of four antihistamine drugs diphenhydramine, chlorpheniramine, cyproheptadine and fexofenadine in pharmaceutical forms by a single HPLC method. Int $J$ Pharm Pharm Sci. 2018;10(4):53. doi:10.22159/ ijpps.2018v10i4.24819

13. Arefin P, Hasan I, Islam MS, Reza MS. Formulation and In vitro Evaluation of Eudragit RL 100 Loaded Fexofenadine HCl Microspheres. Bangladesh Pharm J. 2016;19(1):58-67. doi:10.3329/bpj.v19i1.29240

14. Arefin P, Habib MS, Ahmed NU, et al. Microspheres and microcapsules: A review of their manufacturing techniques for Pharmaceutical industries. Indian J Nov Drug Deliv. 2020;12(4):177-185.

15. Arefin P, Habib MS, Chakraborty D, Bhattacharjee SC, Das S. An overview of microcapsule dosage form. Int J Pharm Chem Anal. 2020;7(4):155160. doi:10.18231/j.ijpca.2020.025

16. Wang J, Cheng X, Li Y, You J. Microsphere with narrow nanopores: Fabrication in PVDF/PMMA/ PLLA blend and enhanced adsorption/separation performances. Appl Surf Sci. 2021;566:150673. doi:10.1016/j.apsusc.2021.150673

17. Wenzhi S, Dezhou W, Min G, Chunyu H, Lanlan Z, Peibiao Z. Assessment of nano-hydroxyapatite and poly (lactide-co-glycolide) nanocomposite microspheres fabricated by novel airflow shearing technique for in vivo bone repair. Mater Sci Eng C. 2021;128:112299. doi:10.1016/j. msec.2021.112299

18. Arefin P, Habib MS, Mostafa M, et al. Evaluation of the Influence of Stirring Speed on the Release Kinetics of Fexofenadine $\mathrm{HCl}$ Polymeric
Microspheres. Biosci Biotechnol Res Asia. 2021;18(4).

19. Arefin P, Ahmed R, Habib MS, et al. Evaluation of Critical Quality Attributes of Immediate Release Ciprofloxacin Tablets of Different Pharmaceutical Companies in Bangladesh. Biosci Biotechnol Res Asia. 2021;17(4):781-788. doi:10.13005/bbra/2883

20. Oktemer T, Altintoprak N, Muluk NB, et al. Clinical Efficacy of Immunotherapy in Allergic Rhinitis. Am J Rhinol Allergy. 2016; 30(5 suppl):S4-S7. doi:10.2500/ajra.2016.30.4368

21. Hossenbaccus L, Linton S, Garvey S, Ellis AK. Towards definitive management of allergic rhinitis: best use of new and established therapies. Allergy, Asthma Clin Immunol. 2020;16(1):39. doi:10.1186/s13223-020-00436-y

22. Baraniuk JN. Pathogenesis of allergic rhinitis. Am J Rhinol. 1997;11(3):245.

23. Skoner DP. Allergic rhinitis: Definition, epidemiology, pathophysiology, detection, and diagnosis. J Allergy Clin Immunol. $2001 ; 108(1):$ S 2-S 8. doi: $10.1067 /$ mai.2001.115569

24. Mir E, Panjabi C, Shah A. Impact of allergic rhinitis in school going children. Asia Pac Allergy. 2012;2(2):93. doi:10.5415/apallergy.2012.2.2.93

25. Moote W, Kim H, Ellis AK. Allergen-specific immunotherapy. Allergy, Asthma Clin Immunol. 2018;14(S2):53. doi:10.1186/s13223-018-02825

26. Gu Y, Liu Y, Cao X. Evolving strategies for tumor immunotherapy: Enhancing the enhancer and suppressing the suppressor. Natl Sci Rev. 2017; 4(2):161-163. doi:10.1093/nsr/nwx032

27. Ellis AK, Gagnon R, Hammerby E, Lau A. Sublingual immunotherapy tablet for the treatment of house dust mite allergic rhinitis in Canada: an alternative to minimize treatment costs? Allergy, Asthma Clin Immunol. 2019;15(1):27. doi:10.1186/s13223-019-0344-3

28. Bernstein DI, Bardelas JA, Svanholm Fogh B, Kaur A, Li Z, Nolte H. A practical guide to the sublingual immunotherapy tablet adverse event profile: implications for clinical practice. Postgrad Med. 2017;129(6):590-597. doi:10.10 80/00325481.2017.1302306

29. Wahn U, Bachert C, Heinrich J, Richter H, Zielen $\mathrm{S}$. Real-world benefits of allergen immunotherapy for birch pollen-associated allergic rhinitis and asthma. Allergy. 2019;74(3):594-604. doi:10.1111/all.13598

30. Sur DK, Scandale S. Treatment of allergic rhinitis. Am Fam Physician. 2010;81(12):14401446. doi:10.4102/safp.v52i5.1656

31. Khan NU, Begum KS. Allergic Rhinitis during 
Pregnancy -an Update of Management. Med Today. 2017;28(2):83-88. doi:10.3329/medtoday. v28i2.32933

32. Petersen TH, Agertoft L. Corticosteroids for Allergic Rhinitis. Curr Treat Options Allergy. 2016;3(1):18-30. doi:10.1007/s40521-016-00753

33. Varshney J, Varshney H. Allergic rhinitis: An overview. Indian J Otolaryngol Head Neck Surg. 2015;67(2):143-149. doi:10.1007/s12070-0150828-5

34. Titulaer J, Arefian H, Hartmann M, Younis MZ, Guntinas-Lichius O. Cost-effectiveness of allergic rhinitis treatment: An exploratory study. SAGE Open Med. 2018;6:205031211879458. doi:10.1177/2050312118794588

35. Kim H, Bouchard J, Renzi PM. The link between allergic rhinitis and asthma: A role for antileukotrienes? Can Respir J. 2008;15(2):9198. doi:10.1155/2008/416095

36. Juel-Berg N, Darling P, Bolvig J, et al. Intranasal Corticosteroids Compared with Oral Antihistamines in Allergic Rhinitis: A Systematic Review and Meta-Analysis. Am J Rhinol Allergy. 2017;31(1):e19-e28. doi:10.2500/ ajra.2016.30.4397
37. Hox V, Lourijsen E, Jordens A, et al. Benefits and harm of systemic steroids for short- And longterm use in rhinitis and rhinosinusitis: An EAACI position paper. Clin Transl Allergy. 2020;10(1):127. doi:10.1186/s13601-019-0303-6

38. Nadel JA. Modulation of neurogenic inflammation by peptidases. Neuropeptides Respir Med. 2017;372(5):351-372. doi:10.1201/9780203745915

39. Valovirta E, Petersen TH, Piotrowska T, et al. Results from the 5-year SQ grass sublingual immunotherapy tablet asthma prevention (GAP) trial in children with grass pollen allergy. $J$ Allergy Clin Immunol. 2018;141(2):529-538. e13. doi:10.1016/j.jaci.2017.06.014

40. Okubo K, Suzuki T, Tanaka A, Aoki H. Efficacy and safety of rupatadine in Japanese patients with seasonal allergic rhinitis: A double-blind, randomized, multicenter, placebo-controlled clinical trial. Allergol Int. 2019;68(2):207-215. doi:10.1016/j.alit.2018.08.011

41. Church M, Church D. Pharmacology of antihistamines. Indian $J$ Dermatol. 2013;58(3):219. doi:10.4103/0019-5154.110832 